\title{
Comparison of FEM Simulations for the Incremental Forming Process
}

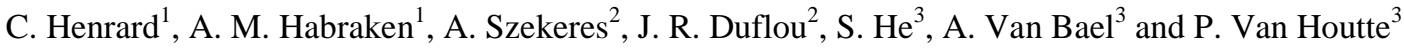 \\ ${ }^{I}$ Department of Mechanics of Solids and Materials (M\&S) - Université de Liège Chemin des Chevreuils 1 - 4000 Liège - Belgium \\ ${ }^{2}$ Department of Mechanical Engineering (PMA) - Katholieke Universiteit Leuven Celestijnenlaan 300A - 3001 Heverlee - Belgium \\ ${ }^{3}$ Department of Metallurgy and Materials Engineering (MTM) - Katholieke Universiteit Leuven Kasteelpark Arenberg 44 - 3001 Heverlee - \\ Belgium
}

\begin{abstract}
Incremental forming is an innovative and highly flexible sheet metal forming technology for small batch production and prototyping that does not require any adapted dies or punches to form a complex shape. The purpose of this article is to perform FEM simulations of the forming of a cone with a 50-degree wall angle by incremental forming and to investigate the influence of some crucial computational parameters on the simulation. The influence of several parameters will be discussed: the FEM code used (Abaqus or Lagamine, a code developed at the University of Liège), the mesh size, the potential simplification due to the symmetry of the part and the friction coefficient. The output is given in terms of final geometry (which depends on the springback), strain history and distribution during the deformation, as well as reaction forces. It will be shown that the deformation is localized around the tool and that the deformations constantly remain close to a plane strain state for this geometry. Moreover, the tool reaction clearly depends on the way the contact is taken into account.
\end{abstract}

Keywords: finite element method, identification, incremental forming.

\section{Introduction}

The classical sheet metal forming process is deep drawing. This process is highly efficient as far as accuracy and speed are concerned but requires the manufacturing of adapted punches and dies, which are very expensive. Consequently, it can only be profitable if a very large number of parts are needed.

Incremental forming is a rather new sheet metal forming process and was developed in response to the observed deficiency in rapid prototyping in this field. It uses a standard smooth-end tool mounted on a numerically controlled multi-axis milling machine. This tool follows a complex tool-path and progressively deforms a clamped sheet into its desired shape.

It has been shown in literature that the formability of sheet metal is higher in incremental forming [1-3]. A deformation state that exceeds the forming limit curve in conventional forming according to the Forming Limit Diagram (FLD) can indeed be reached. The purpose of this article is to analyze the local behavior of the material and to investigate the influence of some relevant parameters.

The subject of the finite element modeling was a cone with a 50-degree wall angle, using the finite element code Lagamine developed at the University of Liège. Some comparisons were also made with the code Abaqus. Since the process is long and requires a fine mesh everywhere in the sheet metal, it would have been appropriate to use shell elements. However, considering that an accurate study of the material state was needed, Kirchhoff or Mindlin's assumption used in shell elements was not suitable. The element chosen for Lagamine simulations was an eight-node mixed type brick element with one integration point called BWD using the new approach for suppressing locking and hourglassing developed by Wagoner et al. [4]. It is an improvement on the BLZ element [5]. The contact element used is the CFI3D based on a penalty approach expressed at integration points [6].

After some preliminary tests, it was found that three layers of brick elements along the thickness were needed in order to acquire accurate results for both the force and the displacement.

Only implicit simulations were performed with both codes in order to obtain stable and accurate results that could be considered as a reference for further investigations.

\section{Benchmark problem}

As a preliminary study, a simple case was simulated for which an analytical solution is available, i.e. the elastic deformation of a simply supported circular plate by a punctual force at its center. As the same mesh density will be applied throughout this article, the purpose of this study was to check the stiffness of the type of elements 
used in that case and validate further simulations.

The equation relating the force $F$ and the displacement of the center of the plate $h$, derived from Kirchhoff's plate theory [7], is:

$$
F=\frac{16 \pi D(v+1)}{r^{2}(v+3)} \cdot h,
$$

with $D=E e^{3} / 12\left(1-v^{2}\right), E=$ Young's modulus, $v=$ Poisson's ratio, $e=$ thickness and $r=$ radius.

For an aluminum alloy AA3003 plate $(E=70000$ [Mpa], $v=0.33), 1.2 \mathrm{~mm}$ thick with a radius of $93 \mathrm{~mm}$, the curves obtained with Abaqus and Lagamine, as well as the theoretical result, are plotted in Fig. 1. Only onefourth of the plate was simulated and the obtained force was multiplied by four.

Fig. 1: Benchmark problem
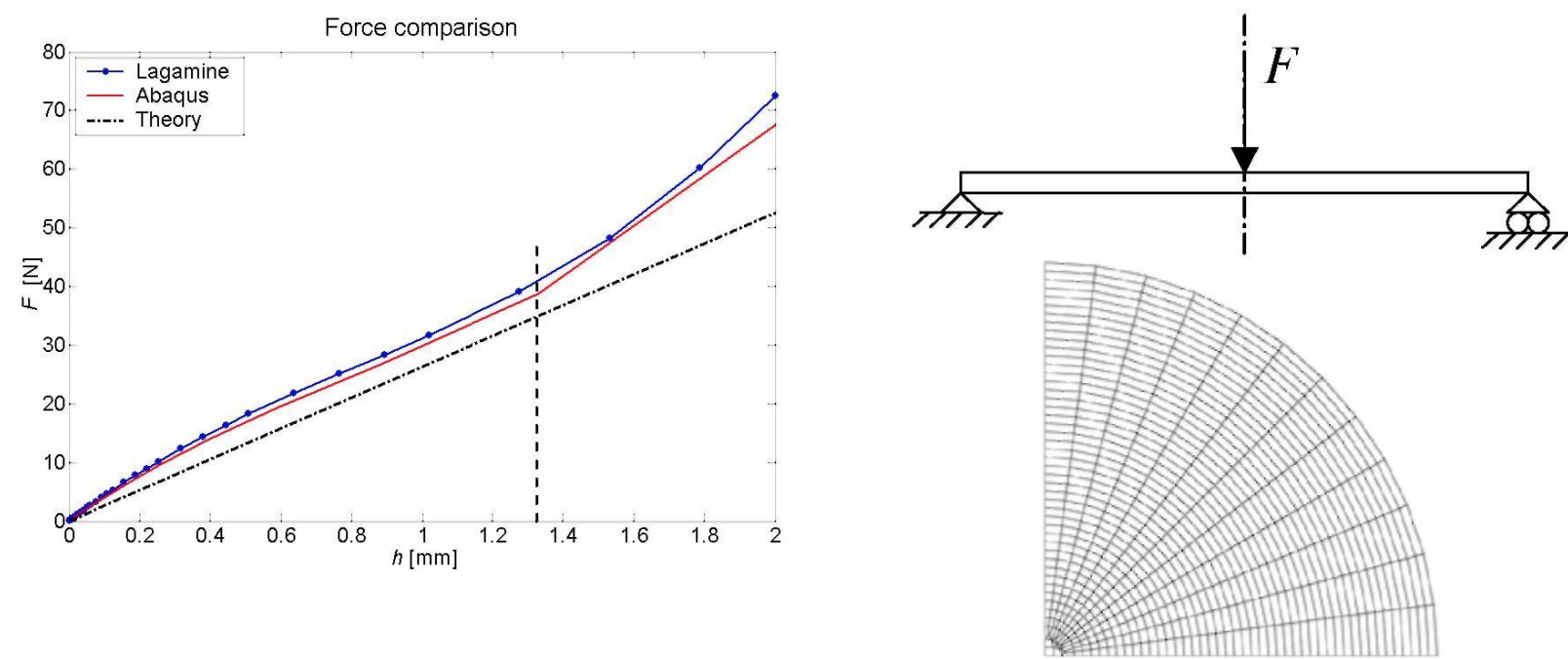

The results obtained with Abaqus and Lagamine are quite similar. In the beginning, both curves are close to the theoretical result. They are highly dependent on the mesh used, especially in the center of the plate. The finer the mesh, the closer the numerical results are to the theory. The simulated curves start diverging from the theory at a displacement of $1.3 \mathrm{~mm}$. This is due to the fact that the theoretical curve neglects the membrane effect and this assumption is no longer valid for bigger displacements, even though the deformation remains purely elastic.

\section{Finite Element Modeling}

From this point on, the simulations of a truncated cone with a 50-degree wall angle will be studied. The same material is used as for the benchmark problem. The flow stress curve obtained by performing a tensile test on a material sample is approximated by

$$
\sigma=180 \cdot(\varepsilon+0.00109)^{0.21}[\mathrm{MPa}]
$$

An anisotropic Hill law was used with Hill coefficients fitted according to measured Lankford coefficients $\left(r_{0}=\right.$ $0.68, r_{45}=0.73$ and $\left.r_{90}=0.66\right)$. The Coulomb's friction coefficient was 0.15 .

The final shape is $40 \mathrm{~mm}$ deep and the tool has a radius of $6.35 \mathrm{~mm}$, as represented in Fig. 2. The tool path is composed of 80 concentric circles, starting from the outermost, with a vertical pitch of $0.5 \mathrm{~mm}$ between them. 
Fig. 2: Cone with $50^{\circ}$ wall angle

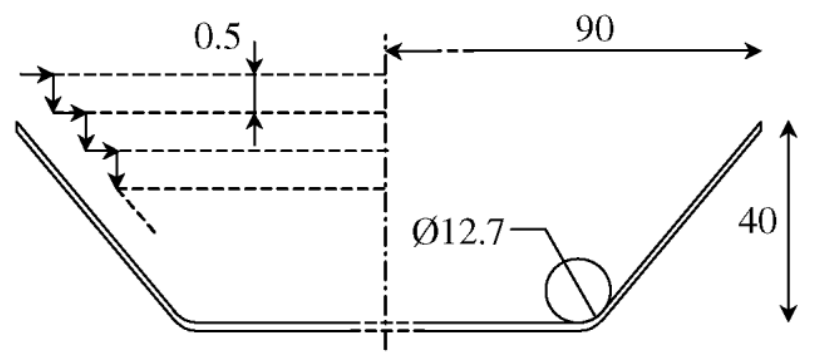

Fig. 3: Initial mesh

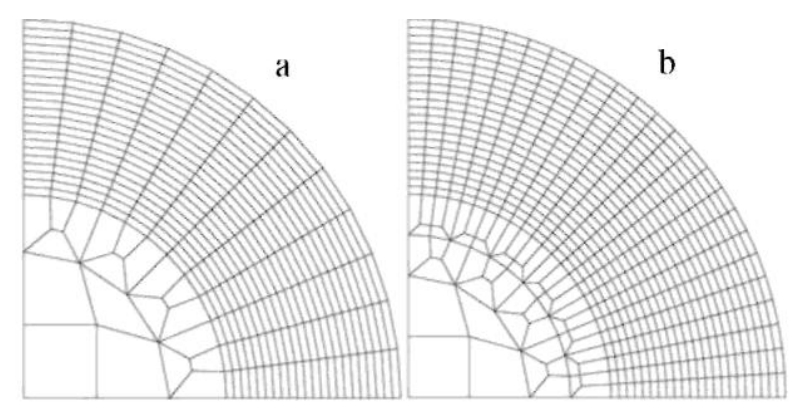

\section{Influence of the mesh and the boundary conditions}

Two different mesh densities were tested: 12 and 24 elements for 90 degrees in the circumferential direction, as shown in Fig. 3. For both meshes, the zone with a higher mesh density starts at a radius $r=50 \mathrm{~mm}$.

Even though the cone is axisymmetrical, the deformation process is not so, because the tool moves around the plate. The purpose of this section is to verify if only one-quarter of the cone can be accurately modeled (after applying proper boundary conditions that replace the rest of the material) by comparing it to the global simulation. Only the region far enough from the boundaries is considered as accurate, i.e. around $45^{\circ}$ from both borders. After some preliminary tests on the type of boundary conditions, it was found that a good choice is to impose that the nodes at the boundaries must stay in their initial vertical planes during the deformation.

The simulation of the whole cone is time-consuming, even with the coarser mesh ${ }^{1}$. Therefore, only the first 20 arcs were simulated instead of 80 . The results are presented hereafter.

\section{Shape and thickness predictions.}

Using Lagamine code, the shape of the cone after 20 arcs has been compared for three different meshes, i.e. a 90degree pie with coarse and fine mesh, and 360 degrees with coarse mesh. The shape and the thickness in a section of the cone are presented in Fig. 4.

The influence of the mesh density is small as far as the shape is concerned. The simplification due to the symmetry of the part appears to be valid in the steep region whereas, in the flat, undeformed region, the boundary conditions do not fully take into account the surrounding material.

\footnotetext{
${ }^{1}$ It takes 15 to 20 hours per arc on a 8 CPU machine (MIPS R12000 at 400MHz) with the Lagamine code
} 
Fig. 4: Comparison of shapes and thicknesses with Lagamine code
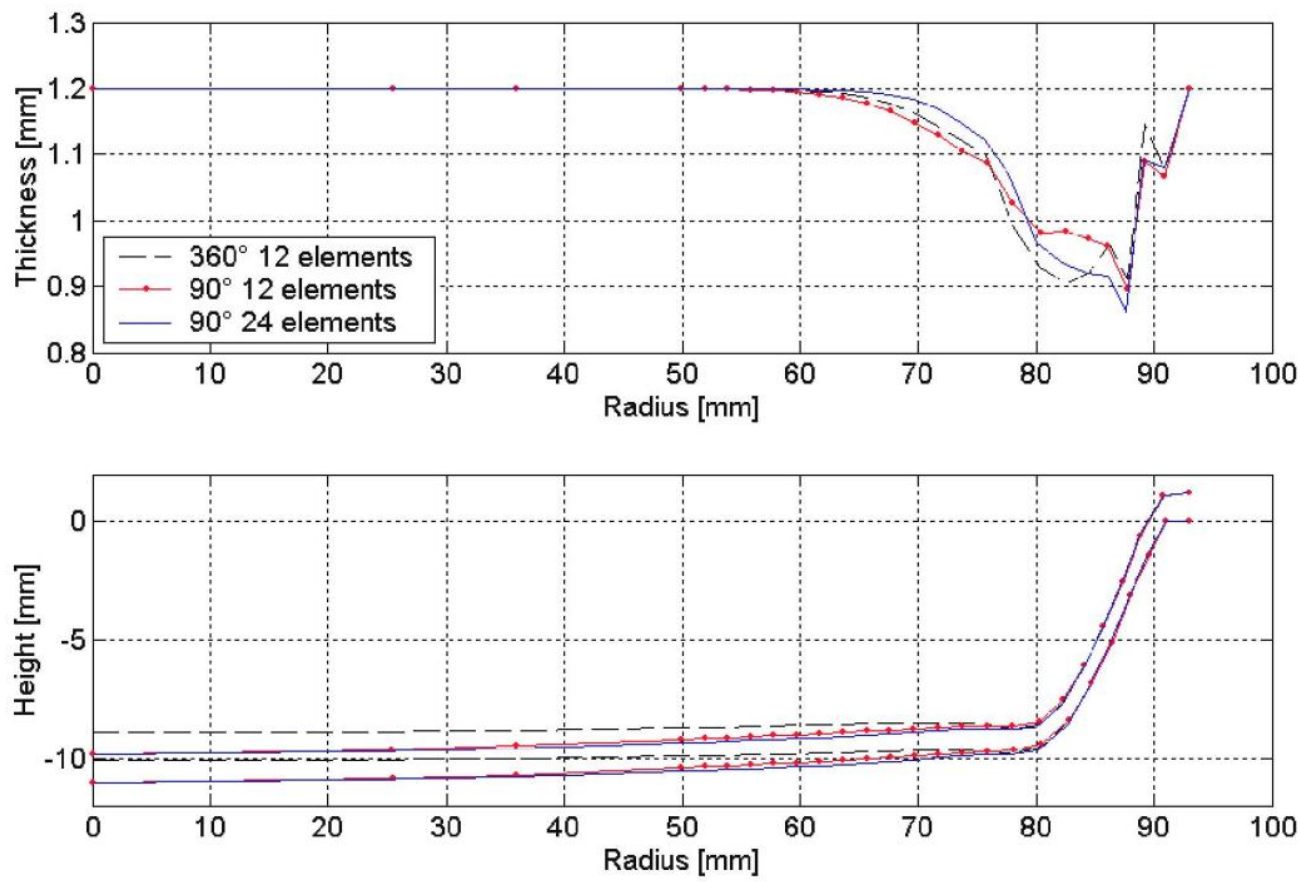

Fig. 5: Comparison of shapes with Abaqus code

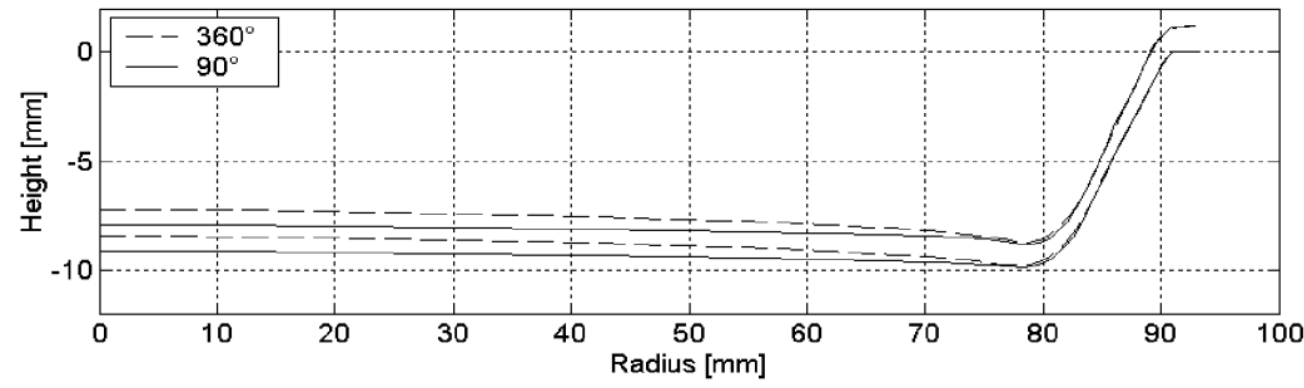

Fig. 6: Vertical force on the tool for the $11^{\text {th }}$ arc
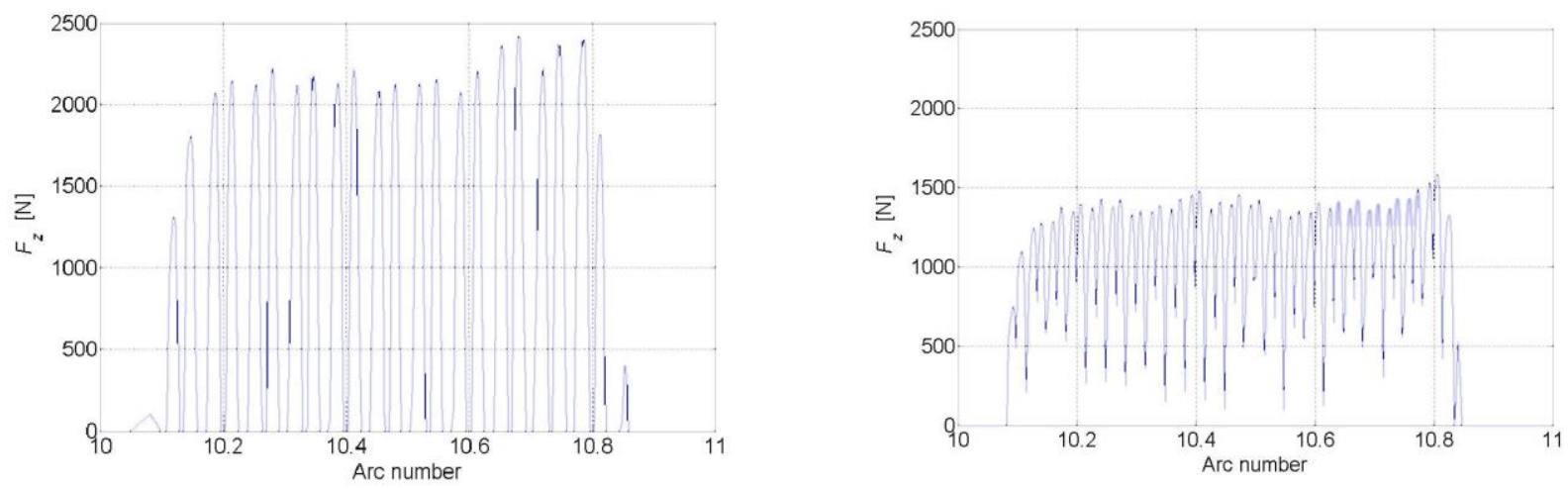

The 360-degree simulation is then about $1 \mathrm{~mm}$ higher in the center of the cone than those of 90 degrees.

Similar simulations were performed with Abaqus using an isotropic von Mises law and a friction coefficient of 0.05. The results are presented in Fig. 5. The same conclusions may be drawn here: the boundary conditions are not completely satisfactory in the flat region. When simulating the whole cone, the center portion is about $1 \mathrm{~mm}$ 
higher than the center of the partial cone.

Even though the simulations done with both codes are not exactly the same (using a different material law and friction coefficient), an important difference should be noted: the plate goes down in the center with Lagamine whereas it goes up with Abaqus. This is due to the fact that the mesh size was twice as big in the radial direction with Lagamine. This causes a greater stiffness in the mesh, which prevents the plate from bending enough under the tool. Further investigations with a finer mesh will be carried out. The comparison of these results with experimental ones [8] shows a center part even higher than Abaqus. Kinematic hardening, which is known to improve springback prediction, will be investigated in order to try to reach a closer agreement with experiments.

\section{Force prediction.}

The influence of the mesh density and the boundary conditions on the vertical reaction force on the tool will now be examined. This result is presented in Fig. 6 for the $11^{\text {th }}$ arc of the 90-degree pie using the two different mesh densities given in Fig. 3. The oscillations in the force depend exclusively on the mesh density. If it is too coarse, the force drops to zero between the integration points of the contact elements; if it is finer, the curve is smoother. In order to get a better idea of the true value of the force, an average must be computed. A good formula for doing so is

$$
F_{\text {avg }}(t)=\frac{1}{t} \cdot \int_{0}^{t} F(s) d s
$$

Using this equation, the average vertical force has been plotted for the three different meshes: the 90-degree pies with coarse and fine mesh, and 360 degrees with coarse mesh, as presented in Fig. 7.

Fig. 7: Average vertical force on the tool

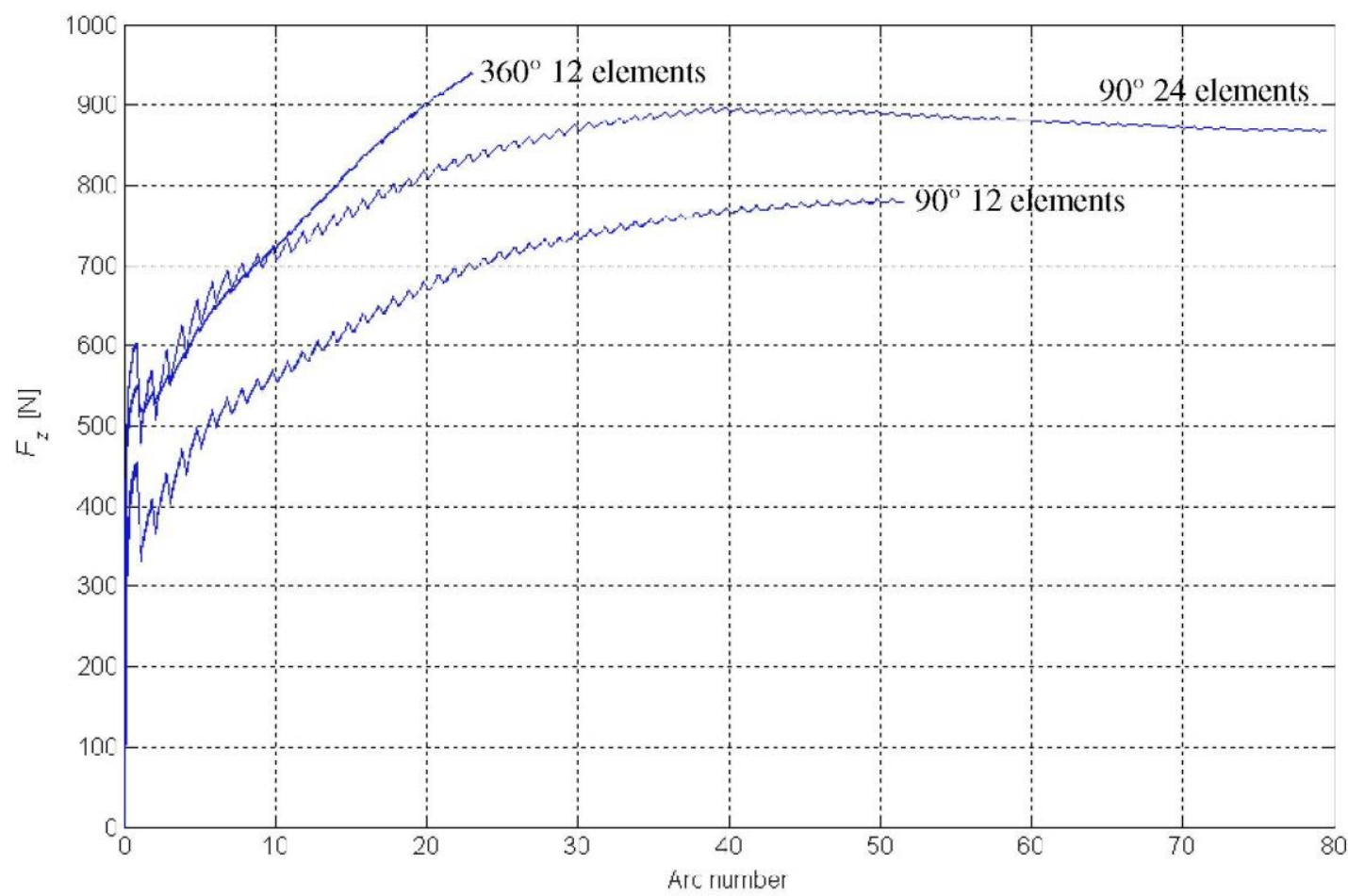

The difference between the three curves is substantial. The force depends to a great extent on the mesh and on the boundary conditions. In order to get accurate and stable results for the force, a very fine mesh and a complete model of the cone must be used, which is not feasible in a reasonable computation time without major modifications to the finite element code. 


\section{Analysis of strain state and history}

The evolution of the three principal strains for element 3 in Fig. 9a is presented in Fig. 8. The second principal strain remains constantly near zero. It corresponds in reality to a plane strain state, as stated in the literature [1,2]. It is the component in the circumferential direction that vanishes, i.e. the component in the direction of the tool movement.

The equivalent strain is represented for several elements in Fig. 9a and b. In the radial direction, the tool influences the equivalent strain of element 3 between the $25^{\text {th }}$ and $69^{\text {th }}$ arc.

Fig. 8: Principal strains

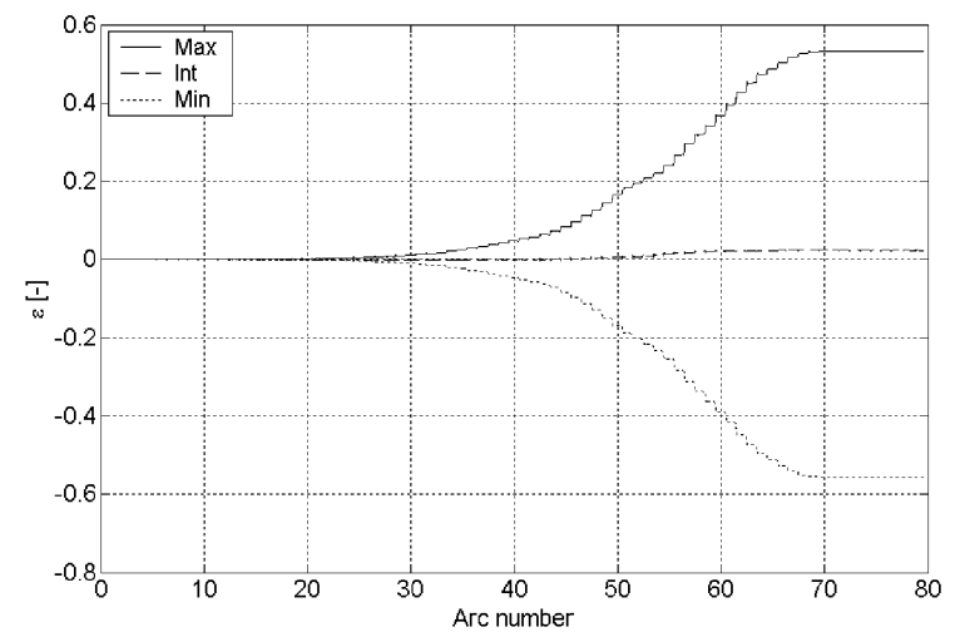

Fig. 9a: Selection of elements in the top layer

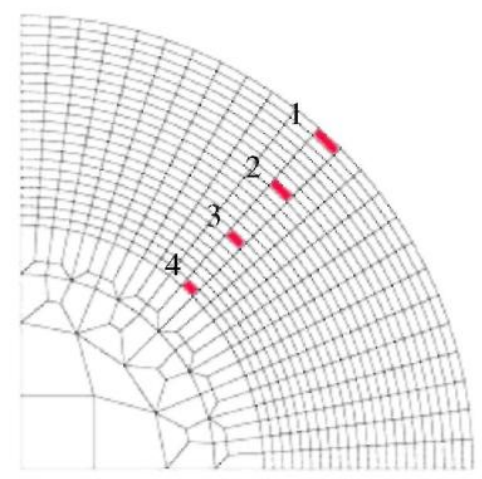

Fig. 9b: Equivalent strain

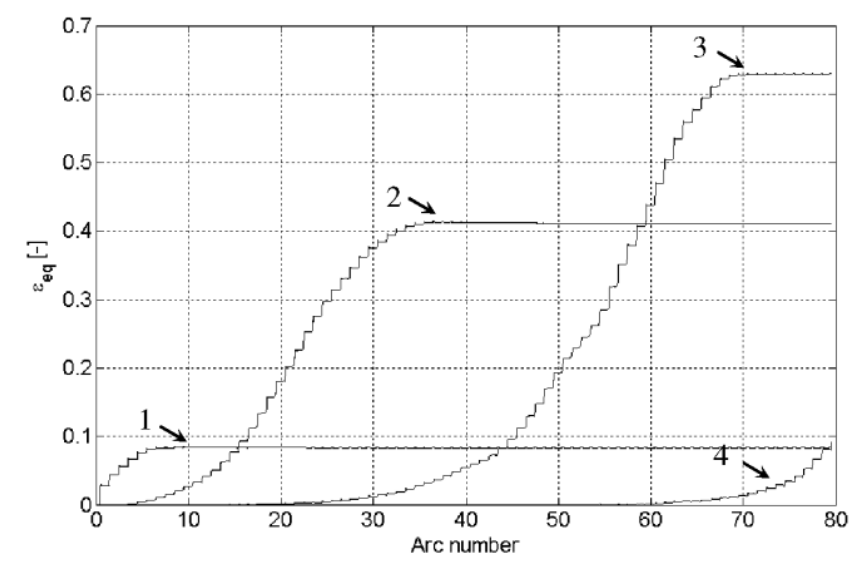


This influence corresponds to a $29 \mathrm{~mm}$ length along the 50-degree slope ${ }^{2}$. The dimension of the zone of influence in the radial direction is then approximately equal to the tool diameter $(12.7 \mathrm{~mm})$ before and after the tool position. The same analysis has been done in the circumferential direction only considering one arc. The zone of influence in that case is about one-half of the tool diameter.

The influence of the tool is then well-localized: the area farther than one tool-diameter in any direction is not subjected to deformation.

\section{Effect of the friction coefficient}

The influence of the friction coefficient on the total force on the tool, i.e. on

$$
F=\sqrt{F_{x}^{2}+F_{y}^{2}+F_{z}^{2}}
$$

will now be analyzed. Two simulations were performed with a 90-degree pie and the coarse mesh, using two different friction coefficients: $\phi=0.05$ and $\phi=0.15$. The results are presented in Fig. 10. The total force is slightly higher $(4 \%)$ with the larger friction coefficient.

Fig. 10: Influence of the friction coefficient on the total force

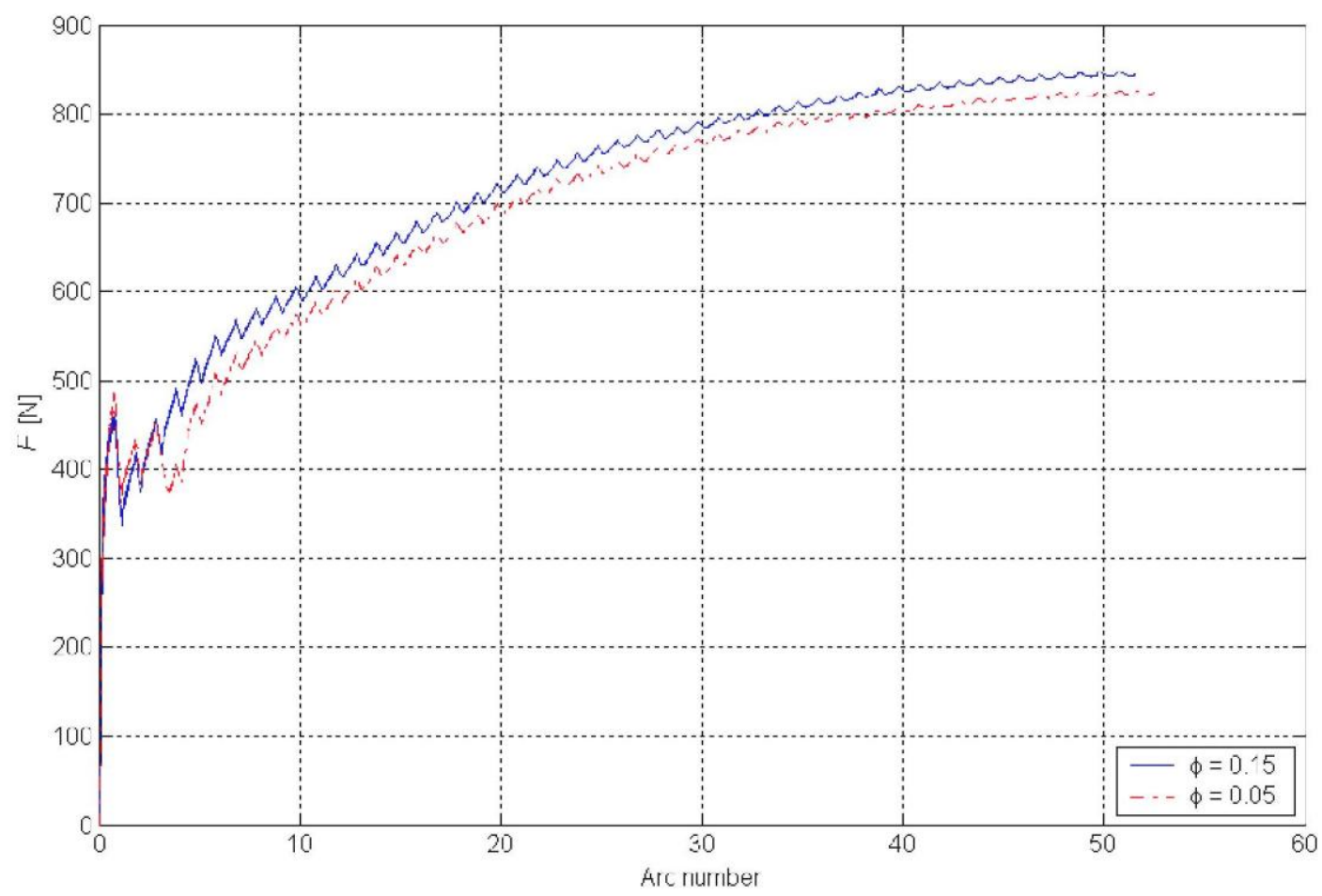

\section{Conclusions}

In this article, the influence of several numerical parameters on the simulation of the incremental forming of a 50-degree wall angle cone was analyzed. Comparisons with experiments are shown in another article presented in the same conference [8].

The global shape and the reaction force on the tool, as well as the local state of the material around the tool, were examined. The mesh density in the circumferential direction has little influence on the final shape, whereas it has a substantial influence on the force. The radial mesh density and whether or not a kinematic hardening is used, are suspected to be responsible for the inaccurate prediction of the geometry of the bottom of the cone. The simplification due to the symmetry of the cone significantly influences both the shape and the force and hence

${ }^{2} d=(69-25) \cdot 0.5 / \sin 50 \approx 29 \mathrm{~mm}$ 
should not be used.

Throughout this article, it was shown that the mesh density plays an important role in the quality of the results. This reinforces the idea that modeling the whole process of incremental forming with a conventional finite element code is almost impossible within a reasonable computation time. That is the reason why a new explicit shell approach is being developed, taking advantage of the fact that the contact is limited to a small number of elements. This research will be presented in another paper soon.

\section{Acknowledgements}

The authors would like to acknowledge the financial support from the Institute for the Promotion of Innovation by Science and Technology in Flanders (IWT).

As Senior Research Associate of the National Fund for Scientific Research (Belgium), A.M. Habraken thanks this Belgian research foundation for its support.

\section{References}

[1] Y.H. Kim and J.J. Park: Journal of Materials Processing Technology Vol. 130-131 (2002), pp. $42-46$

[2] G. Hirt, S. Junk and N. Witulski: Proceedings of the 7th ICTP Vol. 1 (2002), pp. 925-930

[3] G. Ambrogio, L. Filice, L. Fratini and F. Micari: Proceedings of the 6th ESAFORM conference Vol. 1 (2003), pp. 175-178

[4] J. Wang and R. Wagoner: Proceedings of the 8th NUMIFORM conference (2004), pp. 2181-2186

[5] Y.Y. Zhu and S. Cescotto: Computers and Structures, Vol. 53-2 (1994), pp. 275-304

[6] A.M. Habraken and S. Cescotto: Mathematical and Computer Modeling, Vol. 28-4,8 (1998), pp. 153-169

[7] S. Timoshenko and S. Woinowski-Krieger: Theory of Plates and Shells (McGraw-Hill, 2nd Edition, 1953)

[8] S. He, A. Van Bael, P. Van Houtte, A. Szekeres, J.R. Duflou, C. Henrard and A.M. Habraken: Proceedings of the 11th SheMet conference (2005) 